\title{
Similia similibus curentur - a novel treatment approach in endocrinology?
}

\author{
Regina Streuli ${ }^{1 / 2}$, Lea Slahor ${ }^{2}$, Lukas Burget ${ }^{2}$, Christoph Henzen ${ }^{2}$, Stefan Fischli
}

${ }^{1}$ Departement of Medicine, Division of Endocrinology, Diabetes, Osteology and Metabolism, Kantonsspital St. Gallen, CH-9007 St. Gallen ${ }^{2}$ Departement of Medicine, Division of Endocrinology, Diabetes and Clinical Nutrition, Luzerner Kantonsspital, CH-6000 Luzern 16

\section{Background}

Hyperinsulinemic hypoglycemia after gastric bypass surgery is a rare but probably underestimated complication. The mechanisms leading to hypoglycemia are complex and incompletely understood and involve several factors: A part from decreasing insulin resistance, caloric restriction and alteration of nutrient delivery, enhanced secretion of insulin and incretins (GLP-1 and GIP), altered physiology of other hormones (i.e. PYY, Ghrelin, adipokines) and dysregulation of counterregulatory effectors (i.e. glucagon) seem to play a major role. Diagnostic steps involve usually the reproduction of the Whipple triad and the biochemical documentation of endogenous hyperinsulinism in a mixed-meal test. In addition, continuous glucose monitoring system (CGMS) can be helpful for detection of low glucose values after meals and for identification of patients at risk. Treatment strategies include nutritional counselling with carbohydrate restriction and various medical treatments (acarbose, diazoxide, calcium-channel blockers, octreotide, pasireotide, sirolimus). Partial pancreatectomy is reserved for severe treatment-refractory cases. Newer publications suggest the modification of the incretin pathway in these patients either by stimulation [1] or blockage [2] of the GLP1 receptor.

\section{Case report}

A 53-year-old woman presented with postprandial hypoglycemia and neuroglycopenia 3 years after Roux-en-Y gastric bypass. A CGMS over one week and SMBG demonstrated several hypoglycemic episodes typically occurring 2 to 3 hours after meals (Fig. 1/3). Endogenous hyperinsulinism was documented in a mixed meal test which was stopped after 2 hours when the patient had severe neuroglycopenic symptoms and a plasma glucose of $1.9 \mathrm{mmol} / \mathrm{l}$ (Tab. 1). ${ }^{18} \mathrm{~F}$-DOPA-PET showed diffuse uptake within the pancreas but no focal enhancement (Fig. 2). Despite carbohydrate restriction the patient continued to have neuroglycopenic symptoms. Diazoxide was begun but had to be withdrawn due to side effects. After discussion of other treatment options, the patient was started on liraglutide (Victoza ${ }^{\circledR}$ ) which was titrated to a dose of $1.2 \mathrm{mg}$ daily. Frequency and severity of postprandial hyperinsulinemic hypoglycemia decreased markedly and neuroglycopenia resolved completely (Fig. 4). Tolerance of the medication is excellent and no side effects are noted.

Fig. 1 CGMS, demonstrating hypoglycemic episodes after meals.

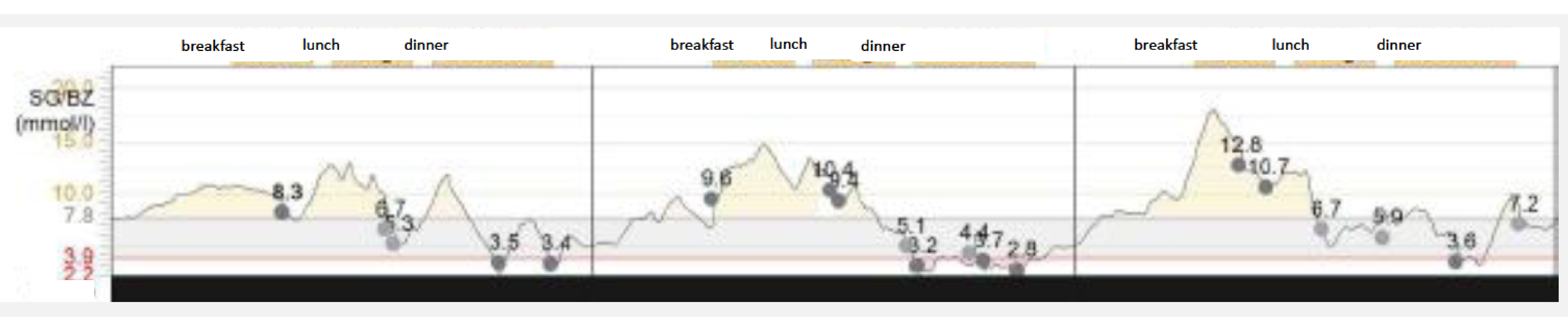

\begin{tabular}{|l|c|c|c|c|c|c|}
\hline $\begin{array}{l}\text { Time (min.) } \\
\text { after meal }\end{array}$ & 0 & 60 & 120 & 150 & 180 & 240 \\
\hline $\begin{array}{l}\text { Plasma glucose } \\
\text { [mmol/L] }\end{array}$ & 4.6 & 9.6 & 4.0 & 1.9 & 5.5 & 5.1 \\
\hline C-Peptide [pmol/L] & 339 & 1892 & 1896 & 968 & 821 & 379 \\
\hline Insulin [mU/L] & 3.6 & 139.1 & 18.1 & 3.4 & 3.7 & 3.0 \\
\hline
\end{tabular}

Tab. 1 Mixed meal test. The test was stopped after $150 \mathrm{~min}$. The patient showed severe neuroglycopenia and a plasma glucose of $1.9 \mathrm{mmol} / \mathrm{l}$.

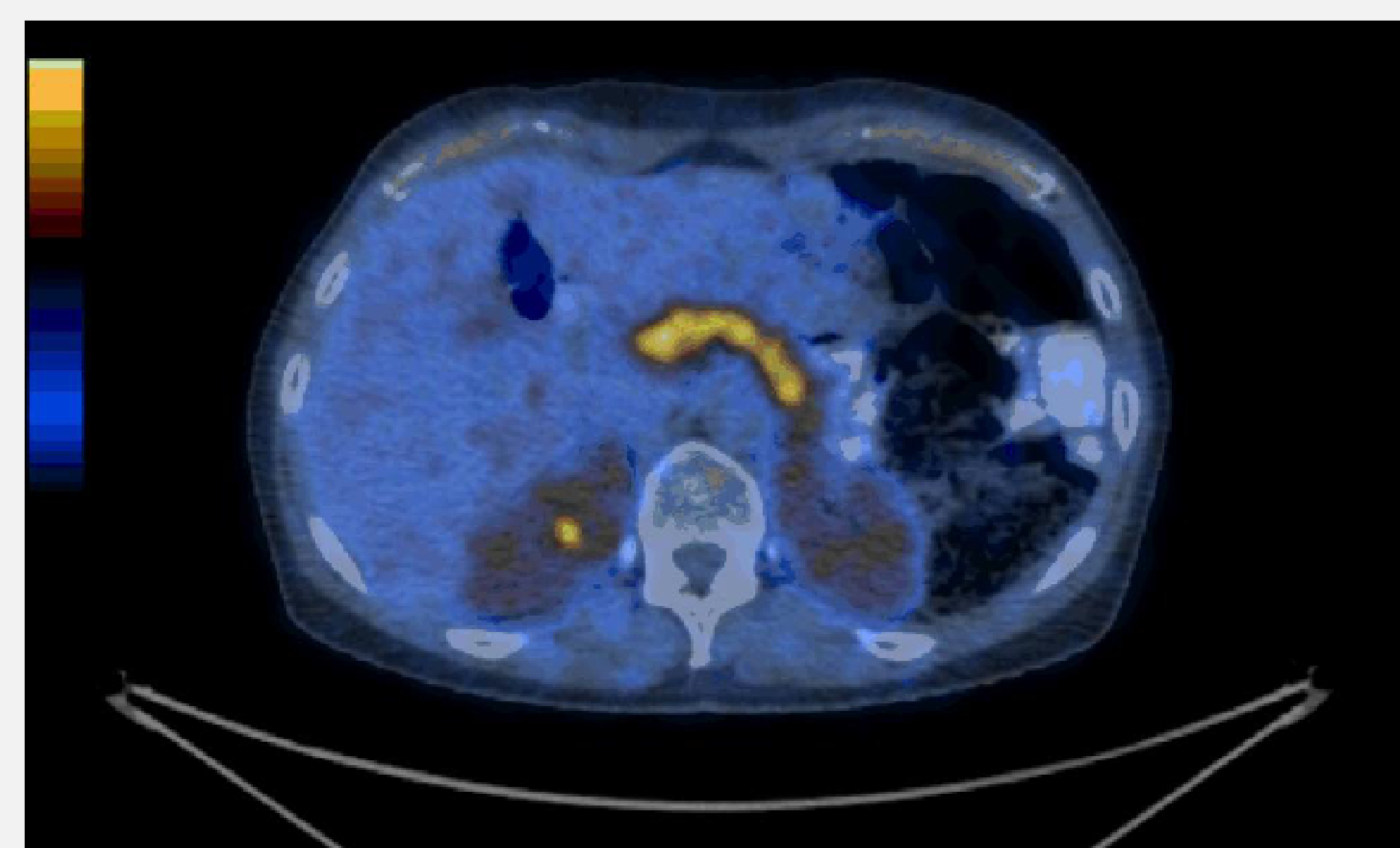

Fig. $2 \square{ }^{18} \mathrm{~F}$-DOPA-PET showing diffuse uptake within the pancreas but no focal lesion.

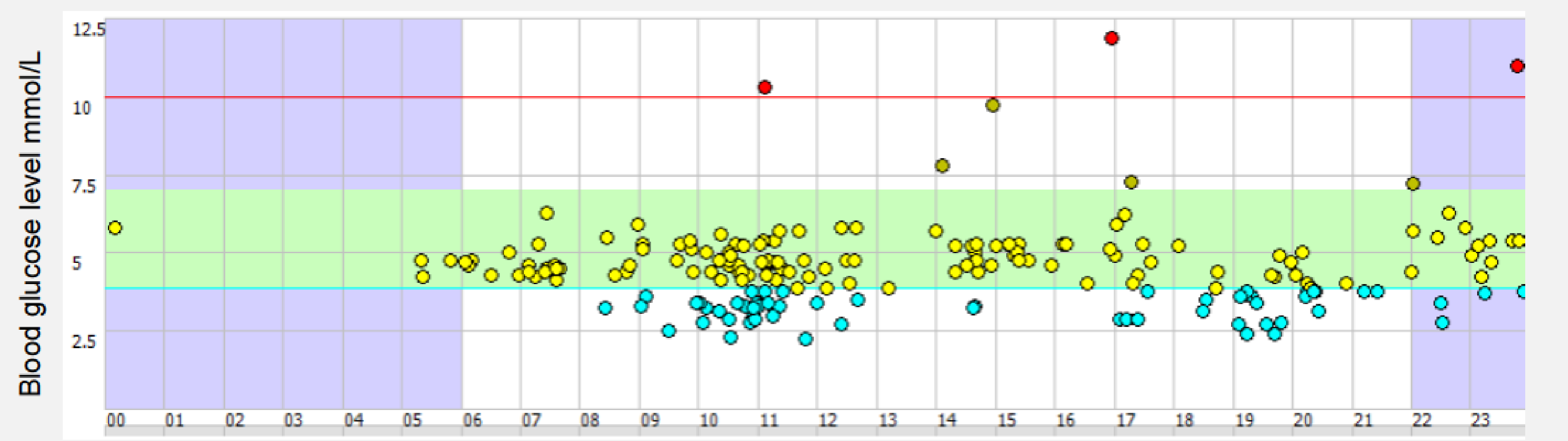

Fig. $3 \square$ SMBG: Glucose levels before treatment with liraglutide.

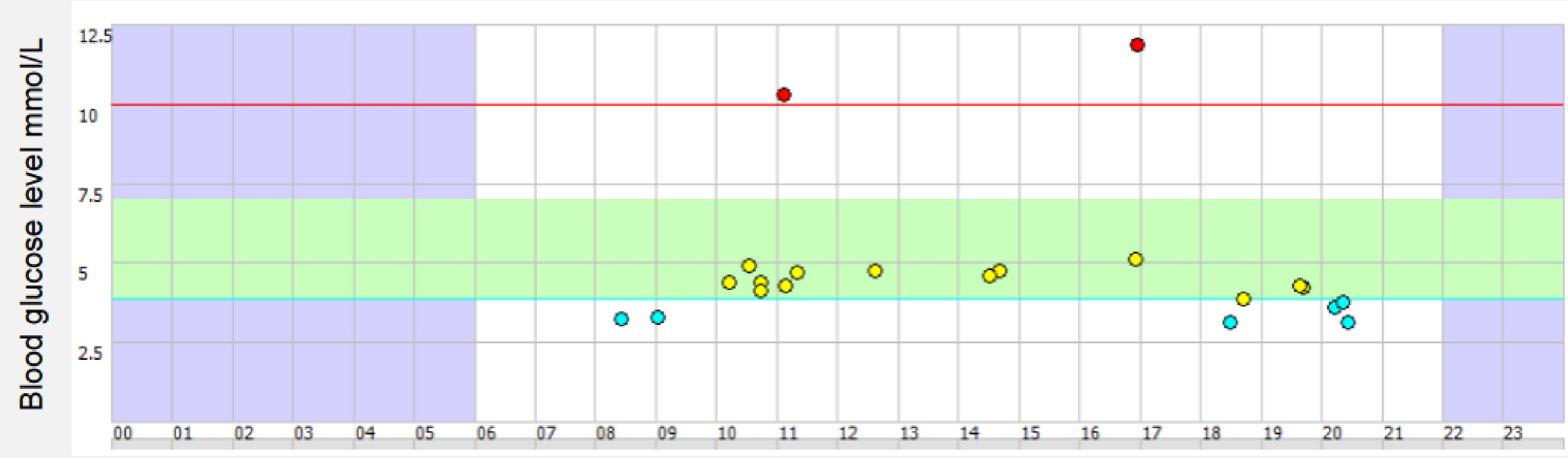

Fig. $4 \square$ SMBG: Glucose levels under treatment with liraglutide.

\section{Conclusions}

GLP-1 analogues might be a new treatment option for hyperinsulinemic hypoglycemia after bariatric surgery. However, the exact mechanism of action remains to be elucidated. It can be assumed that a stimulation of glucagon secretion with concurrent inhibition of insulin secretion in low blood glucose states by GLP-1 analogues partly explains the stabilizing effect on glucose metabolism in these patients. Nevertheless, further studies are needed.

References

1. Abrahamsson, N., Engstrom, B. E., Sundbom, M. \& Karlsson, F. A. GLP1 analogs as treatment of postprandial hypoglycemia following gastric bypass surgery: a potential new indication? Eur J Endocrinol 169, 885-889 (2013).

2. Salehi, M., Gastaldelli, A. \& D’Alessio, D. A. Blockade of glucagon-like peptide 1 receptor corrects postprandial hypoglycemia after gastric bypass. Gastroenterology 146, 669680.e2 (2014). 